\title{
Propuesta de documento de voluntades anticipadas específico para pacientes con insuficiencia renal crónica
}

\author{
Begoña Capillas Echevarría, Zuriñe Itxaso Gil García, Magdalena Berasategui Burguera, Yolanda Martínez \\ Martínez, Iñaki Saralegi Reta, Arantza Arrausi Larrea
}

Hospital Universitario de Álava. Santiago. Álava

\section{Introducción:}

Las Voluntades Anticipadas son procesos de comunicación y toma de decisiones compartidas que incluyen, personal sanitario, pacientes y familiares. Se recomienda especialmente para planificar la asistencia sanitaria en la fase final de la vida en pacientes crónicos, como los pacientes con Insuficiencia renal crónica en diálisis (IRC-D). Aunque en general los pacientes con IRC-D se muestran partidarios de redactar un Documento de Voluntades Anticipadas (DVA) específico que recoja sus preferencias para que sean tomadas en cuenta cuando no sean competentes, la mayoría desconoce el proceso de planificación y no existen programas específicos para pacientes IRC-D.

\section{Objetivo:}

Elaborar un DVA específico para pacientes IRC-D como resultado del conocimiento de sus preferencias y su valoración de la calidad de vida relacionada con la salud.

\section{Método:}

Para conocer las vivencias de los pacientes y que este conocimiento contribuyera a adecuar los procesos de planificación y que los DVA recojan las preferencias de los pacientes se realizó un estudio cualitativo. Este estudio diseñado como investigación-acción participativa contaba con dos entrevistas semiestructuradas a pacientes IRC-D. Se llevó a cabo un muestreo intencional con un tamaño de muestra definido por el criterio de saturación. De mayo a diciembre de 2012 se entrevistaron a 14 pacientes IRC-D, acompañados de algún familiar si lo deseaban: nueve hombres y cinco mujeres, la mitad de ellos en hemodiálisis y la otra mitad con diálisis peritoneal. Las entrevistas fueron grabadas con el permiso del paciente para su posterior análisis de contenido. Para elaborar el documento se partió del análisis de la información del estudio anterior y se creó un grupo de trabajo multidisciplinar integrado por profesionales de hemodiálisis e investigadores de toma de decisiones al final de la vida. Se partió de los modelos de DVA existentes, y se incorporó a estos modelos la perspectiva de los pacientes con IRC-D.

\section{Resultados:}

El documento de VA final consta de una parte inicial en la que se describe los deseos de los pacientes. En esta parte se manifiesta cómo se desea mantener la diálisis como soporte vital mientras puedan mantener una aceptable calidad de vida, que para ellos significa poder comunicarse y realizar las actividades básicas de la vida diaria, además de no tener dolor o sufrimiento. En una segunda parte, se especifica las situaciones en que se decide suspender el tratamiento de diálisis o el no comienzo de otro tratamiento que prolongue el proceso de morir, como sería padecer una condición incurable o irreversible sin expectativas de recuperación (situación terminal, inconsciencia permanente). En estos casos, el documento expresa el deseo de evitar el dolor y el sufrimiento y de ser cuidado a ser posible en el domicilio, salvo que suponga una carga excesiva para los familiares del paciente. 


\section{Conclusiones:}

EI DVA específico propuesto para pacientes IRC-D recoge los aspectos más relevantes para los pacientes entrevistados. Conocer las vivencias de los pacientes contribuye a adecuar los procesos de planificación de la atención al final de la vida y a elaborar DVA que recojan sus preferencias.

\section{Referencias Bibliográficas}

1. Alcázar R, Egocheaga MI, Orte L, et al. Documento de consenso SEN-semFYC sobre la enfermedad renal crónica. Nefrología 2008;28:273-80.

2. Gibson JL, Upshur RE. Ethics and chronic disease: where are the bioethicists? Bioethics 2012:26:iiiv.

3. De Francisco AL, Sanjuán F, Foraster A, et al. Estudio epidemiológico de pacientes ancianos con insuficiencia renal crónica en hemodiálisis. Nefrología 2008; 28:48-53.

4. Dasgupta I, Rayner HC. In good conscience-safely withholding dialysis in the elderly. Semin Dial 2009;22:476-9.
5. Cohen LM, Germain MJ, Poppel DM. Practical considerations in dialysis withdrawal: "to have that option is a blessing". Jama 2003; 289:2113-9.

6. Murtagh FE, Marsh JE, Donohoe $P$, Ekbal NJ, Sheerin NS, Harris FE. Dialysis or not? A comparative survival study of patients over 75 years with chronic kidney disease stage 5 . Nephrol Dial Transplant 2007;22: 1955-62.

7. Germain MJ, Davison SN, Moss AH. When enough is enough: the nephrologist's responsibility in ordering dialysis treatments. Am J Kidney Dis 2011;58:135-43.

8. Tejedor A, De las Cuevas Bou X. Cuidado paliativo en el paciente con enfermedadcrenal crónica avanzado (grado 5) no susceptible de tratamiento dialítico. Nefrología 2008; Supl. 3:129-36.

9. Seghal AR, Weisheit C, Miura $Y$, Butzlaff M, Kielstein R, Y. T. Advance Directives and Withdrawal of Dialysis in the United States, Germany, and Japan. Jama 1996;276:1652-6.

10. Bartlow B. In search of an advance directive that works for end-stage renal disease patients. Hemodialysis International 2006;10:538-45. 